\title{
PA-141 THE INTERNATIONAL GOOD CLINICAL PRACTICES GUIDELINES: TIME FOR A REVISION?
}

Raffaella Ravinetto, ${ }^{1}$ Halidou Tinto, ${ }^{2}$ Ermias Diro, ${ }^{3}$ Joseph Okebe, ${ }^{4}$ Yodi Mahendradhata, ${ }^{5}$ Suman Rijal, ${ }^{6}$ Eduardo Gotuzzo, ${ }^{7}$ Pascal Lutumba, ${ }^{8}$ Alain Nahum, ${ }^{9}$ Kateljine De Nys, ${ }^{10}$ Minnes Casteels, ${ }^{10}$ Marleen Boelaert ${ }^{1}$. ${ }^{1}$ ITM Antwerp, Belgium; ${ }^{2}$ CRUN, Burkina Faso; ${ }^{3}$ University of Gondar, Ethiopia; ${ }^{4} \mathrm{MRC}$, The Gambia; ${ }^{5}$ Gadiaj Madah University, Indonesia; ${ }^{6}$ BPKIHS, Nepal; ${ }^{7}$ Humboldt Tropical Medicine Institute, Peru; ${ }^{8}$ University of Kinshasa, Republic of Congo; ${ }^{9}$ CREC, Benin; ${ }^{10} \mathrm{KU}$ Leuven, Belgium

\subsection{6/bmjgh-2016-000260.167}

Background The Good Clinical Practices (GCP) codes of the World Health Organization and the International Conference of Harmonization set international standards for clinical research. But critics argue that they were written without considering the challenges faced by clinical researchers in low- and middle-income countries (LMIC).

Methods We analysed the challenges met when conducting clinical trials in LMIC, including in several locations in sub-Saharan Africa and in EDCTP-funded trials. We compared these challenges to GCP guidance, in order to (a) verify if there are gaps between the international GCP codes and the field reality in LMIC, and (b) formulate recommendations for GCP improvement if needed.

Results We identified shortcomings in the GCP guidance concerning three broad domains: ethical, legal and operational. We identified also eleven specific issues: the double ethical review of 'externally sponsored' trials; the informed consent in children; the informed consent in illiterate people; the informed consent comprehension; the definition of vulnerability; the post-trial access to communities; the role of communities as key stakeholders in research; the definition of sponsor; the guidance for contractual agreements; the clinical monitoring; the laboratory quality management systems; and the quality assurance of investigational products. For each specific issue, we formulated a recommendation for the improvement of GCP.

Conclusions Clinical trials are increasingly conducted in LMICs, thus a comprehensive revision of GCP guidelines is needed, to ensure adequate guidance for researchers operating in these contexts, and to maximise protection of research participants. The revised GCP code should be strongly rooted in ethics, sensitive to different socio-cultural perspectives, and allow consideration of trial- and context-specific challenges. This can be only achieved if researchers, sponsors, regulators 
and ethical reviewers from LMIC are transparently involved in the revision process, as well as non-commercial researchers and sponsors, and major agencies that fund international collaborative clinical research. 